\title{
Experimental Testing of Single APM Spheres
}

\author{
M. Vesenjak ${ }^{1, a}$ and L. Krstulovic-Opara ${ }^{2, b}$ \\ ${ }^{1}$ University of Maribor, Faculty of Mechanical Engineering, Maribor, Slovenia \\ ${ }^{2}$ University of Split, Faculty of Elect. Eng., Mech. Eng. and Naval Architect., Split, Croatia
}

\begin{abstract}
Advanced pore morphology (APM) foam, consisting of sphere-like metallic foam elements, proves to have advantageous mechanical properties and unique application adjustability. Since the APM foam manufacturing procedure has been developed recently, the mechanical characterization of these materials is still very limited. Therefore, the purpose of this research was to determine the behaviour of APM spheres and its composites when subjected to quasi-static and dynamic compressive loading. The results of the performed research have shown valuable mechanical properties of the composite APM foam structures, offering new possibilities for their use in general engineering applications.
\end{abstract}

\section{Introduction}

Development, design, manufacturing, characterisation and application of conventional cellular structures used in composite materials have been widely studied and reviewed [1-4]. New fabrication methods have been developed for more convenient and flexible use in different applications to overcome the technological problems related to the control of structure irregularity, also resulting in more homogeneous and regular pore distribution [5-8]. Recently, a new type of cellular materials has been developed, the Advanced Pore Morphology (APM) foam, which consists of sphere-like metallic foam elements, characterised by advantageous mechanical properties and unique applicability. Since the APM foam manufacturing procedure has recently been developed, the mechanical characterization of these materials is very limited. Therefore, the purpose of this research was to determine the behaviour of the APM foam elements subjected to compressive loading. Single APM foam elements were experimentally subjected to quasi-static and dynamic compressive loading, providing the basic properties and knowledge for an efficient application of composite APM foam structures.

\section{Advanced Pore Morphology Foam}

The APM foam has been developed at Fraunhofer IFAM, Bremen in Germany, and represents a new method for production of hybrid cellular structures. The APM foam elements (spheres) consist of interconnected closed-cell cellular structure (foam), as shown in Fig. 1 [9-10].

\footnotetext{
a e-mail : m.vesenjak@uni-mb.si

b e-mail : lovre.krstulovic-opara@fesb.hr
} 

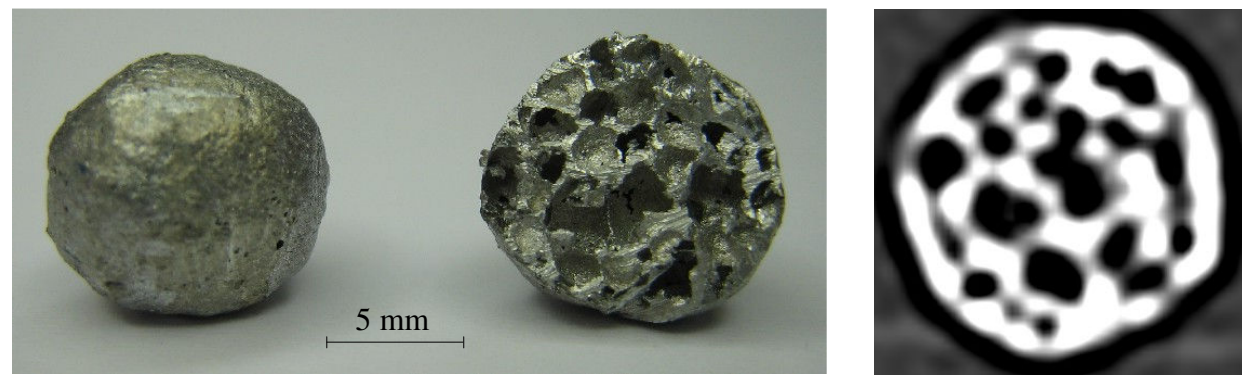

Fig. 1. Advance Pore Morphology (APM) foam element: cross-section (left) and CT scan (right).

The manufacturing procedure consists of powder compaction (by the CONFORM ${ }^{\circledR}$ process) and rolling to obtain expandable precursor material. The precursor material is then cut into small volumes (granules) that are in a continuous belt furnace expanded (due to $\mathrm{TiH} 2$ foaming agent) into spherical foam elements [11]. The matrix alloy of the APM foam is AlSi7. Generally, three sizes of APM foam are manufactured: 5, 10 and $15 \mathrm{~mm}$ in diameter. Their density can vary from 500 to 1000 $\mathrm{kg} / \mathrm{m} 3$ [10]. Their detailed technology concept, production and properties are described in $[9,12]$. The APM foam parts exhibit two types of porosity: (i) the inner porosity in single APM foam elements and (ii) the porosity between the many APM foam elements [13].

As cellular materials, the APM foam elements have a characteristic compressive stress-strain relationship that can be divided into four main areas: (i) quasi-linear elastic response, (ii) transition zone, where the materials exhibit buckling, plastic deformation and collapse of intercellular walls, (iii) stress plateau, where the mechanism of buckling, and collapse becomes even more pronounced, resulting in large strains at almost constant stress and (iv) densification, where the stiffness increases and consequently converges towards the stiffness of the base material. The APM foam has a wide potential application spectrum as energy absorbing structure, stiffening elements (in shell structures), core layers, damping elements or bonded with a matrix in composite materials. One of their main advantages is their simple use as filler elements increasing the energy absorption of hollow parts, e.g. a hollow automotive part can be filled with APM foam elements covered with adhesive, then the filled part is heated up to join the APM foam elements. Such APM foam filled part has a much higher energy absorption capabilities with minimal mass increase. It should be also noted that this procedure is insensitive to hollow part shape and geometry, which can be a challenging problem at conventional aluminium foams. They are additionally able to undergo large deformations when subjected to compressive loading.

Since the technology concept and manufacturing procedure of APM foam elements has just recently been developed there are only a few studies available characterizing this kind of material. The reference [9], along with the detailed description of the APM technology concepts, represents fundamental deformation behaviour of single and bonded APM foam elements under quasi-static compressive loading conditions. Results of uni-axial and hydrostatic compression tests of APM foam are evaluated in [11], where the authors focus on variation and influence of adhesives and adhesive coating thicknesses used for bonding the APM foam elements with partial morphology. Additionally, they provide some distinctive differences between APM and conventional aluminium foams. A practical example of filling a hollow profile with APM elements and comparing the stressstrain diagrams between the hollow profile, hollow profile filled with APM elements and hollow profile filled with bonded APM elements is given in [9, 13]. The authors conclude that filling the profile with APM elements increases the capability of energy absorption which can be further improved by bonding the APM elements. 


\section{Experimental testing}

The experimental testing was divided into two parts: (i) characterization of single APM foam elements and (ii) characterization of composite APM foam. The experiments have been performed on the servo-hydraulic dynamic testing machine INSTRON 8801 under quasi-static and dynamic uni-axial compressive loading conditions (Fig. 2).

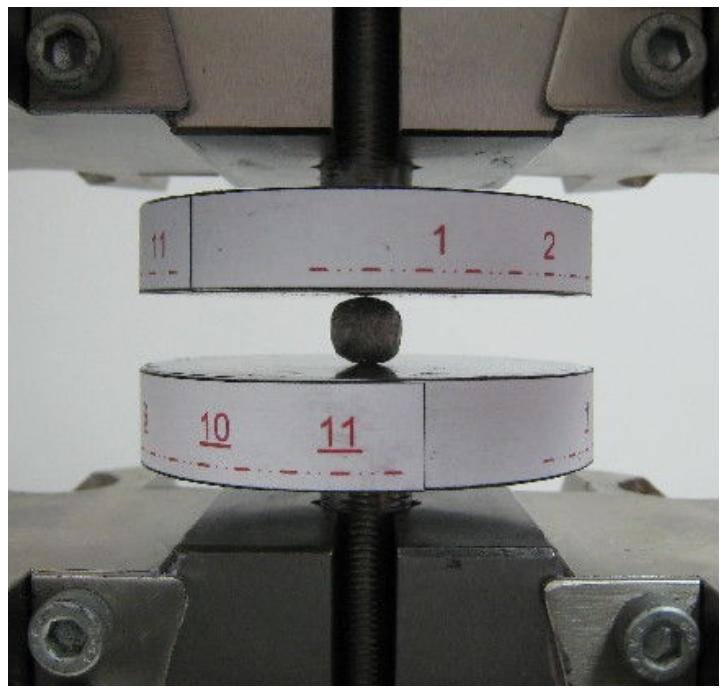

Fig. 2. Compressive testing of a single APM foam element.

Two sizes of APM foam elements $(\rho \approx 800 \mathrm{~kg} / \mathrm{m} 3)$ have been used: $\varnothing 5$ and $\varnothing 10 \mathrm{~mm}$. The matrix alloy of all specimens was AlSi7. Figure 3 shows the deformation behaviour of the APM foam element.

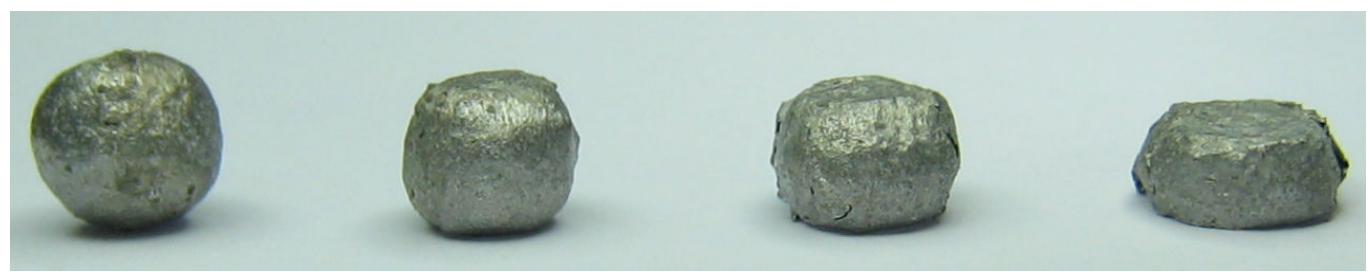

Fig. 3. Compression phases of a single APM foam element.

The results of the single APM foam elements experiments are shown in Fig. 4-8, showing characteristic cellular material (foam) behaviour, i.e. the stress plateau followed by the densification. Due to the spherical shape of the APM foam elements the diagram shows the compressive force vs. macroscopic (global) engineering compressive strain. Figures 4 and 5 represent the quasi-static and dynamic compressive behaviour of APM foam elements of $5 \mathrm{~mm}$ in diameter. Strain rates up to $33 \mathrm{~s}^{-1}$ have been achieved. From the comparison it can be observed that in the case of quasi-static loading the stress increase more gradually compared to the dynamically loaded elements. However, this difference can be observed only up to $15 \%$ of deformation. For the larger deformation, the response of quasi-statically and dynamically loaded APM elements is similar. 


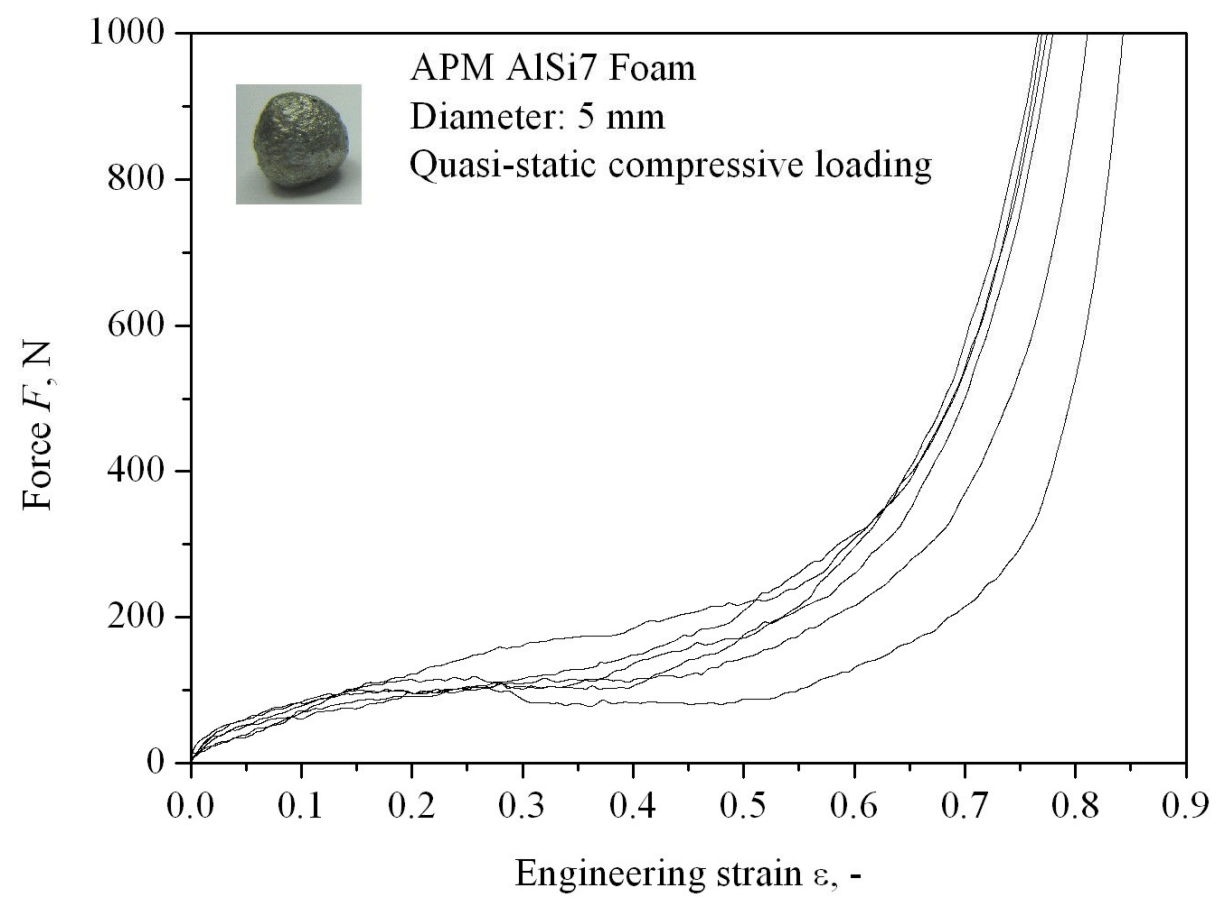

Fig. 4. Behaviour of single APM foam elements $(\varnothing 5 \mathrm{~mm})$ under quasi-static compressive loading conditions.

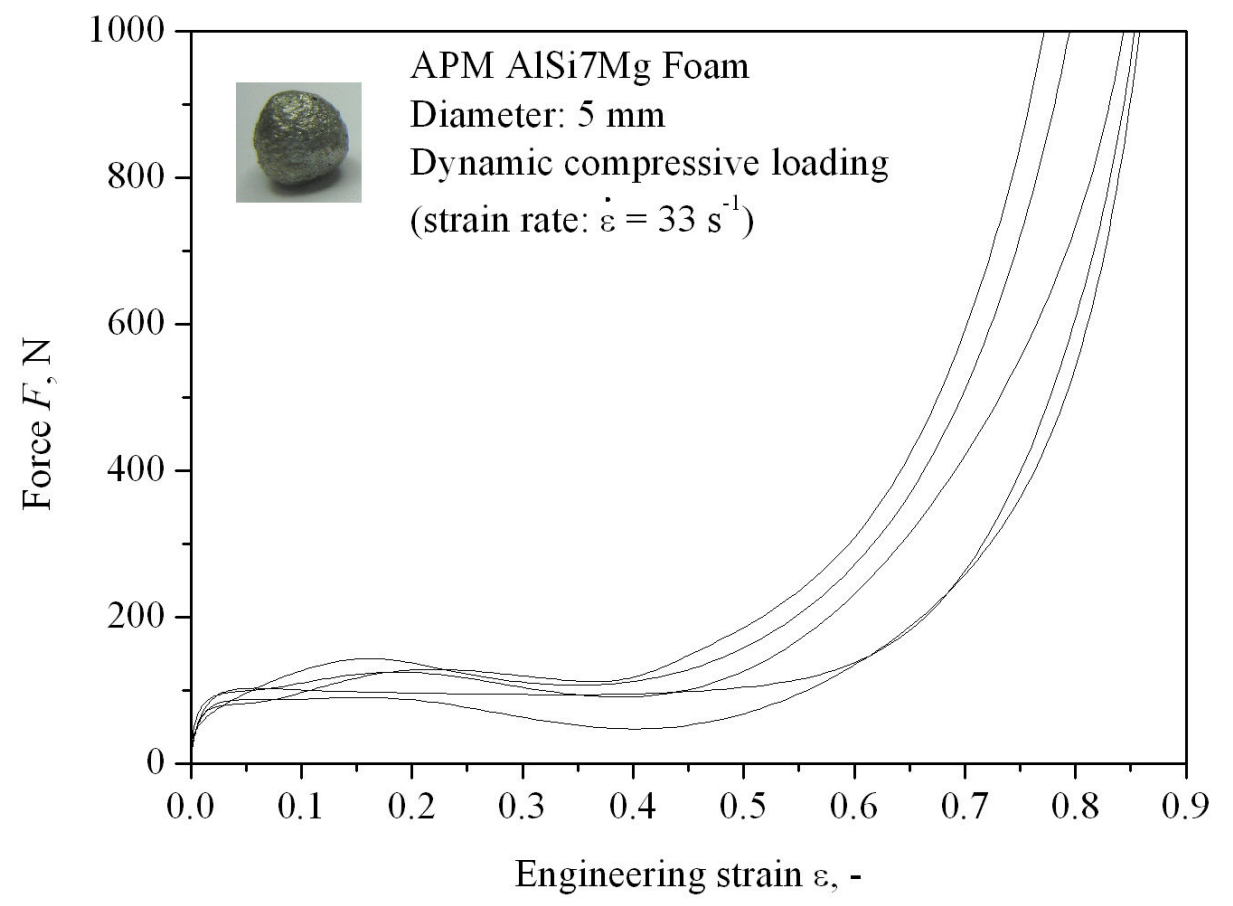

Fig. 5. Behaviour of single APM foam elements ( $\varnothing 5 \mathrm{~mm})$ under dynamic compressive loading conditions. 


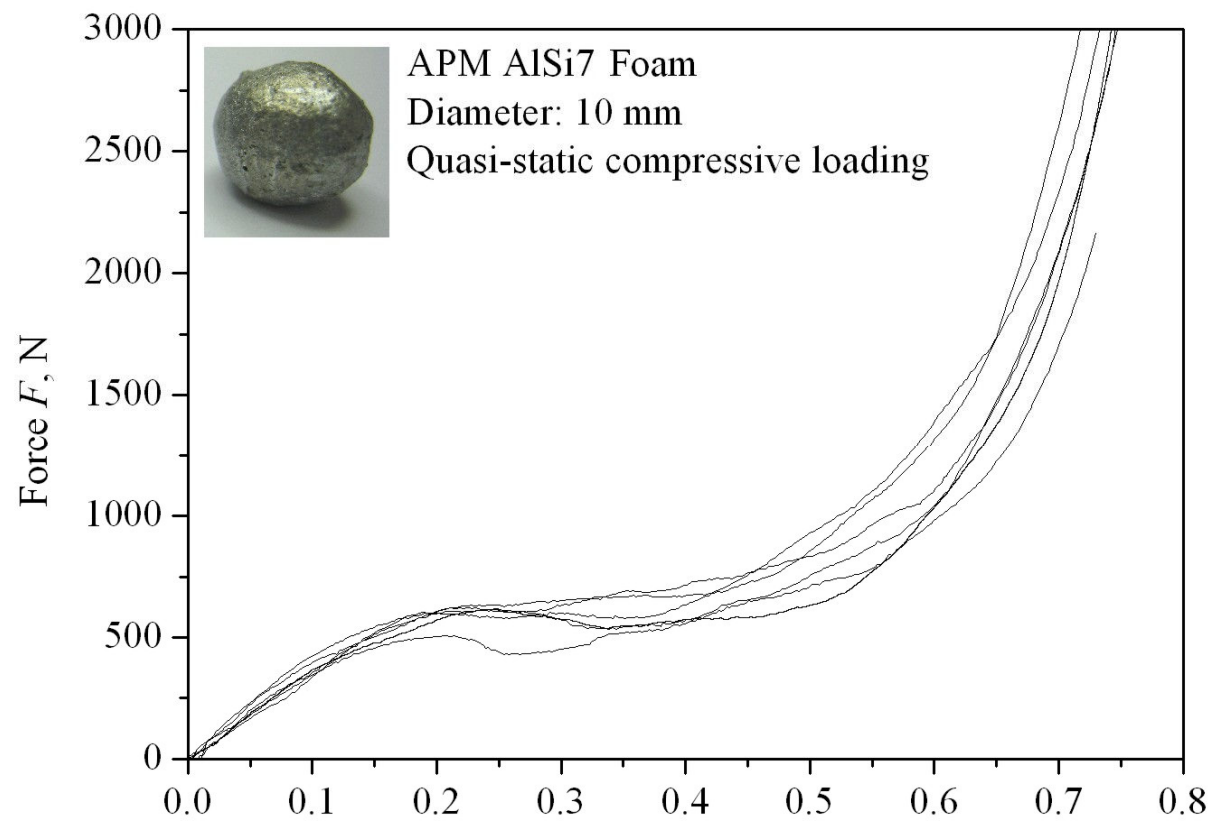

Engineering strain $\varepsilon,-$

Fig. 6. Behaviour of single APM foam elements $(\varnothing 10 \mathrm{~mm})$ under quasi-static compressive loading conditions.

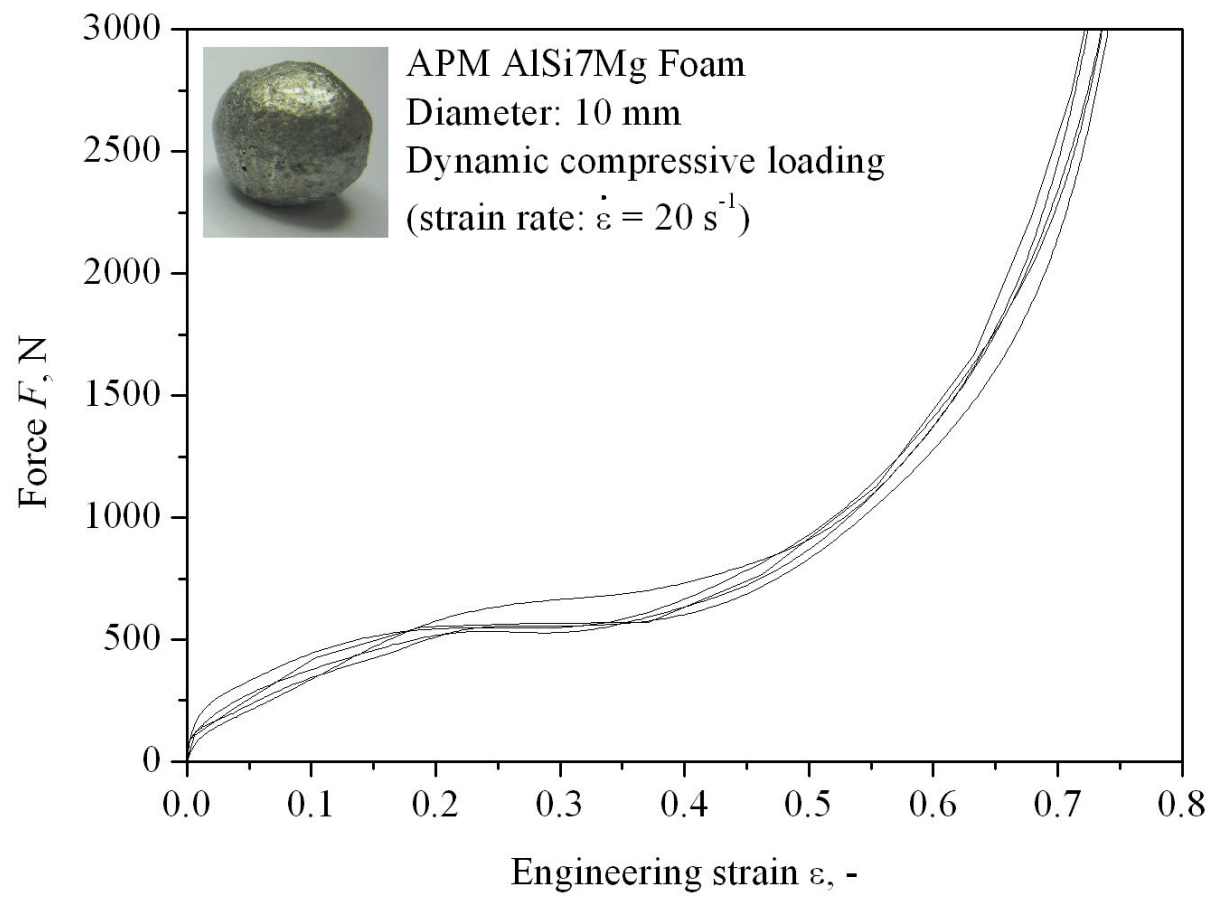

Fig. 7. Behaviour of single APM foam elements $(\varnothing 10 \mathrm{~mm})$ under dynamic compressive loading conditions. 
Figures 6 and 7 represent the quasi-static and dynamic compressive behaviour of APM foam elements with $10 \mathrm{~mm}$ in diameter. Strain rates up to $20 \mathrm{~s}^{-1}$ have been achieved. From the comparison it can be observed that their global response is similar. However, higher strain rates might influence their behaviour.

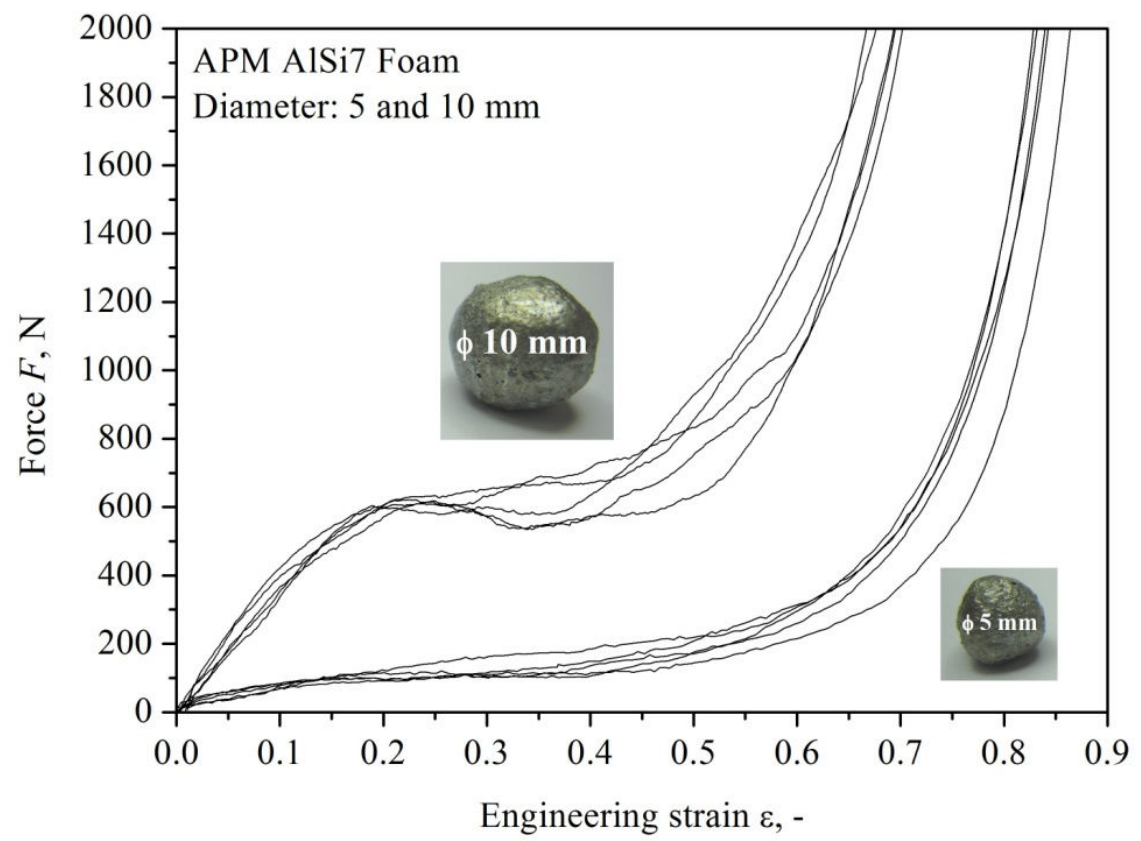

Fig. 8. Behaviour of single APM foam elements $(\varnothing 5$ and $\varnothing 10 \mathrm{~mm})$ under quasi-static compressive loading conditions.

From the Fig. 8 it can be observed that the plateau (force) region for larger specimens $(\varnothing 10 \mathrm{~mm})$ is approximately $4.6 \mathrm{x}$ higher in comparison to $\varnothing 5 \mathrm{~mm}$ APM foam elements. Additionally, it is clear that larger foam elements experience lower densification strain (approx. $\Delta \varepsilon=0.15$ ). The experimental results are in a good agreement with results achieved by Stöbener et al. [11].

\section{Conclusions}

The APM foam elements, characterized by efficient manufacture and repeatable production parameters, are opening new directions in engineering of energy absorption components. The paper describes behaviour of APM foam subjected to compressive mechanical loading. The single APM elements showed a characteristic cellular material behaviour. It has been observed that the larger foam elements experience lower densification strain. Additionally, minor strain rate sensitivity in the global behaviour has been noted. The APM spheres are mainly considered as basic components, which together with adhesive form composite materials having enormous potential in various applications. In our ongoing research an advanced approach to study the topology and morphology influence on the global behaviour of composite APM structures by means of the CT and thermal image scanning is planned. Furthermore, different adhesive materials will be tested in order to increase the application range of the studied composite materials. 


\section{Acknowledgments}

Support of Dr. Karsten Stöbener and Croatian Ministry of Science, Education and Sports project "Fatigue strength of constructions and materials" is gratefully acknowledged.

\section{References}

[1] M.F. Ashby, A. Evans, N.A. Fleck, L.J. Gibson, J.W. Hutchinson, H.N.G. Wadley. Metal foams: a design guide. Burlington, Massachusetts: Elsevier Science (2000).

[2] J. Banhart. Manufacture, characterisation and application of cellular metals and metal foams. Prog Mater Sci, 46, 559 (2001).

[3] L.J. Gibson, M.F. Ashby. Cellular solids: structure and properties. Cambridge: Cambridge University Press (1997).

[4] M. Vesenjak, L. Krstulović-Opara, Z. Ren, A. Öchsner, Ž. Domazet. Experimental Study of Open-Cell Cellular Structures with Elastic Filler Material. Exp Mech, 49, 501 (2009).

[5] O. Andersen, U. Waag, L. Schneider, G. Stephani, B. Kieback. Novel Metallic Hollow Sphere Structures. Adv Eng Mater, 2, 192 (2000).

[6] C. Augustin, W. Hungerbach. Production of hollow spheres (HS) and hollow sphere structures (HSS). Mater Lett, 63, 1109 (2009).

[7] M. Vesenjak, T. Fiedler, Z. Ren, A. Öchsner. Behaviour of Syntactic and Partial Hollow Sphere Structures under Dynamic Loading. Adv Eng Mater, 10, 185 (2008).

[8] H. Nakajima. Fabrication, properties and application of porous metals with directional pores. Prog Mater Sci, 52, 1091 (2007).

[9] K. Stöbener. Advanced Pore Morphology (APM) - Aluminiumschaum. Bremen: University of Bremen (2007).

[10] Advanced Pore Morphology (APM) Metal Foams. Bremen: Fraunhofer Institut Fertigungstechnik Materialforschung (IFAM) (2007).

[11] K. Stöbener, D. Lehmhus, M. Avalle, L. Peroni, M. Busse. Aluminum foam-polymer hybrid structures (APM aluminum foam) in compression testing. Int J Solids Struct, 45, 5627 (2008).

[12] K. Stöbener, G. Rausch. Aluminium foam-polymer composites: processing and characteristics. Journal of Materials Science, 44, 1506 (2009).

[13] K. Stöbener, J. Baumeister, G. Rausch, M. Rausch. Forming metal foams by simpler methods for cheaper solutions. Metal Powder Report, 60, 12 (2005). 